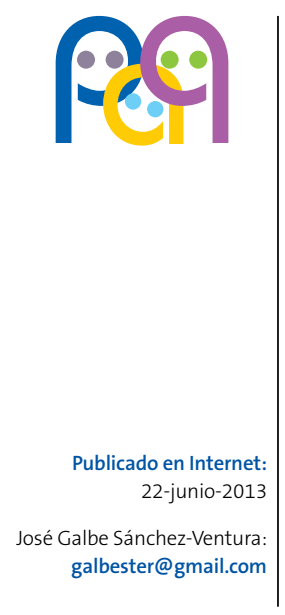

Palabras clave:

- Neumonía atípica

- Mycoplasma

pneumoniae

- Mycobacterium

tuberculosis

- Tuberculosis pulmonar

\title{
Cuando una neumonía atípica es "muy atípica"
}

\author{
R. Fernando Martínez ${ }^{\mathrm{a}}$, C. Fuertes Rodrigo ${ }^{\mathrm{a}}$, G. Gómez Tena ${ }^{\mathrm{a}}$, \\ C. Gargallo Bernad ${ }^{b}$, J. Galbe Sánchez-Ventura ${ }^{c}$
}

\author{
${ }^{a}$ MIR-Pediatría. Hospital Miguel Servet, Zaragoza. España • b MIR-Medicina Familiar y Comunitaria. \\ Hospital Miguel Servet, Zaragoza. España • CPediatra. CS Torrero La Paz, Zaragoza. España.
}

Se presenta el caso de un chico de 14 años que acude a un Servicio de Urgencias hospitalario por presentar dolor mesotorácico y dificultad para respirar. En la radiografía de tórax se aprecia una condensación en el lóbulo superior derecho y serología IgM positiva para Mycoplasma pneumoniae. Inicialmente se diagnosticó de neumonía atípica y se trató con amoxicilina más claritromicina. Cuarenta y ocho horas después acude al centro de Atención Primaria (AP). En la historia clínica de AP figura el antecedente paterno de haber padecido una tuberculosis pleuropulmonar cinco años antes. El niño presentó en ese momento un Mantoux positivo con radiografía de tórax normal y fue tratado de forma profiláctica con isoniacida. Estos antecedentes llevan a plantear el diagnóstico de tuberculosis, repitiéndose el Mantoux y poniendo en marcha la petición de baciloscopias y cultivo para Mycobacterium tuberculosis, siendo ambos positivos. El tratamiento inicial fue sustituido por un tratamiento con cuatro fármacos antituberculosos. La valoración de la renovación de recetas por parte de la enfermera de AP cuestionó el correcto cumplimiento del tratamiento. Todo ello llevó a una mueva intervención del equipo de AP para informar, reforzar y responsabilizar a la familia acerca del correcto cumplimiento del tratamiento.

\section{When atypical pneumonia is "very atypical"}

The authors present the case of a 14 years old boy attended in a hospital emergency room for thoracic pain and difficult breathing. The Rx shows a condensation in LSD and IgM serology positive for mycoplasma pneumoniae. Initially it was diagnosed with atypical pneumonia and was treated with amoxicillin and clarythromicin. Forty-eight hours later he attends the Health Primary Care Center. Primary care medical history contains the paternal antecedent of having suffered a pleuropulmonar tuberculosis five years earlier. The child presented a positive Mantoux with normal Rx at that time and he was treated with prophylactic isoniazid. These records raise the question of the potential diagnosis of tuberculosis, performing the mantoux test again and a smear and culture for mycobacterium tuberculosis, resulting both positive. The initial treatment was replaced by a treatment with four antituberculous drugs. The evaluation of the renewal of prescriptions by the primary care nurse questioned the correct treatment compliance. This led to an intervention of the primary care team to inform, strengthen and force the responsibility of the family about the correct treatment compliance.

\section{INTRODUCCIÓN}

La colaboración entre Atención Primaria (AP) y servicios hospitalarios es fundamental para una atención correcta. A menudo tiende a minusvalorarse el valor de la AP por parte del hospital y viceversa.
El caso que presentamos ilustra perfectamente cómo ante un determinado escenario clínico el diagnóstico y el tratamiento correctos dependen de la aportación de ambos niveles asistenciales. De haberse limitado la atención al nivel primario o al hospitalarion el diagnóstico se hubiera dilatado 
en el tiempo y las deficiencias en el cumplimiento del tratamiento no se hubieran detectado.

\section{CASO CLÍNICO}

F. es un chico de 14 años de origen ecuatoriano, que vive en España desde hace nueve años. Un buen día, durante la primavera, acude directamente al Servicio de Urgencias del hospital pediátrico de referencia por presentar un cuadro de inicio agudo de unas dos horas de evolución de dolor centrotorácico sin fiebre; se acompaña de sensación de "falta de aire". No hay antecedentes traumáticos y lleva unos días tosiendo un poco. Hace tres semanas tuvo un episodio muy similar. En la exploración no se encuentra ninguna alteración. En la radiografía de tórax (Fig. 1) se aprecia una imagen de consolidación en el lóbulo superior derecho. Se realiza el diagnóstico de probable neumonía atípica y se prescribe tratamiento con amoxicilina y claritromicina. Desde el mismo Servicio de Urgencias se solicita una analítica básica y una serología de Mycoplasma pneumoniae. Los resultados estuvieron disponibles en cuatro días; como parámetros a valorar destacan leucocitos

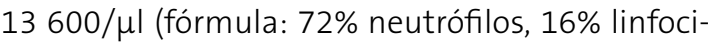
tos y $7,8 \%$ monocitos), velocidad de sedimentación globular $38 \mathrm{~mm}$ a la primera hora; serología de

Figura 1. Radiografía de tórax del paciente, en la que se observa un aumento de densidad en el lóbulo superior derecho

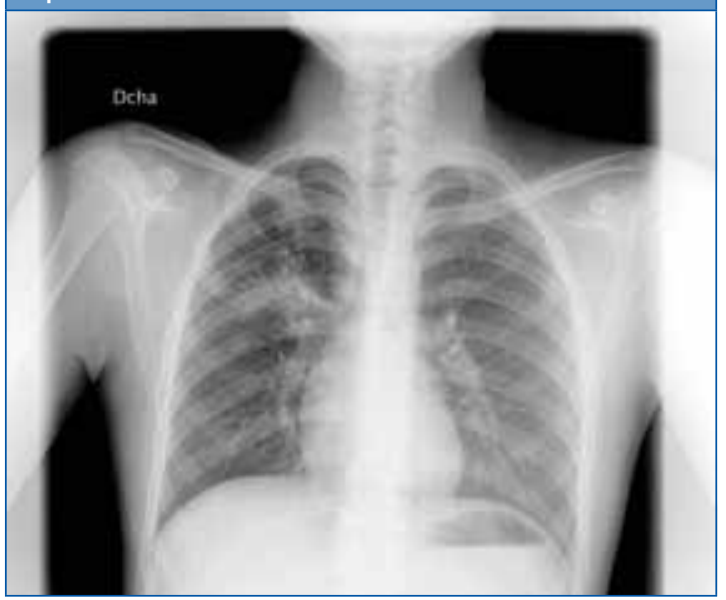

Mycoplasma pneumoniae IgM positiva y proteína C reactiva $4,8 \mathrm{mg} / \mathrm{dl}$.

Dos días después acude con su padre al centro de AP para comentar estos resultados y continuar con el seguimiento. Al pediatra le llama la atención la repetición del cuadro a lo largo de tres semanas, la ausencia de fiebre y la densidad de la imagen radiológica. Al analizar los datos disponibles del niño en la historia clínica, hay que destacar que hace cinco años el padre presentó un cuadro de neumonía tuberculosa acompañada de derrame peural y esputos que resultaron positivos para Mycobacterium tuberculosis. Estos datos y antecedentes no fueron facilitados por el padre al pediatra que le atendió en el Servicio de Urgencias.

En aquel entonces se practicó en el niño una radiografía de tórax que fue normal. La prueba de tuberculina (PTB) mostró una induración de $20 \times 20$ mm con ampolla y esfacelación. El niño había sido vacunado con BCG al nacer. En su momento se prescribió isoniacida, que estuvo tomando durante seis meses sin detectarse fallos en el cumplimiento.

A la vista de estos datos, se decidió repetir la PTB, que volvió a mostrar una induración de $20 \times 20 \mathrm{~mm}$ con ampolla y esfacelación. A continuación se solicitaron tres muestras de jugo gástrico para baciloscopia y cultivo, cuyos resultados se muestran y que fueron idénticos en las tres muestras para ambas técnicas:

- Baciloscopia con tinción de Ziehl-Nielsen: no se observan bacilos ácido-alcohol resistentes, reacción en cadena de la polimerasa (PCR) a Mycobacterium tuberculosis positiva; se realiza la detección de sensibilidad a isoniacida y rifampicina a partir de muestra directa por técnicas moleculares (MTBDR plus) y no se detecta mutación en el gen katG o inhA que codifica la resistencia para isoniacida, lo que se interpreta como sensible aproximadamente en el $80 \%$ de las cepas. Tampoco se detecta mutación para rpoB, que codifica la resistencia a rifampicina, por lo que se interpreta también como sensible en el $90 \%$ de las cepas, por lo que ambos fármacos podrían utilizarse en la pauta de tratamiento. 
- Cultivo de micobacterias: aislamiento a los 12 días de Mycobacterium tuberculosis sensible a estreptomicina, etambutol, isoniacida, pirazinamida y rifampicina.

\section{Tratamiento}

Se pautó tratamiento con isoniacida, pirazinamida, etambutol y rifampicina.

\section{Seguimiento}

Al mes de evolución, el estado general es bueno pero surgen dudas por parte de Enfermería de AP acerca del cumplimiento correcto del tratamiento al evaluar las fechas de renovación de las recetas. Al mismo tiempo, el padre expresa su preocupación por la dieta del niño puesto que come solo, ya que los padres salen muy pronto de casa para trabajar. Este comentario del padre unido a las dudas de la enfermera alertan al pediatra de la posibilidad de incumplimiento del tratamiento, dado que debe ingerir varias pastillas por la mañana.

Se informa al Servicio de Infecciosos del hospital donde también es controlado y se mantiene una nueva entrevista con el padre. El padre manifiesta que tanto él como la madre se van de casa a las 06:00 horas para trabajar, dejando al niño responsable de tomar su medicación. Ante este nuevo dato, insistimos en la necesidad de que le den la medicación al irse y en las posibles consecuencias que tendría no tomar la medicación, desde el punto de vista tanto individual como comunitario y de vigilancia sanitaria.

\section{DISCUSIÓN}

El presente caso ilustra perfectamente un hecho que queremos destacar, como es la necesaria colaboración entre AP y hospitalaria. Cada uno de estos sectores debe aportar valores específicos para proporcionar una asistencia correcta. En este caso, sin esta colaboración el diagnóstico se habría retrasado o no se hubieran detectado los incumplimientos en el tratamiento. Para garantizar esta coordi- nación es importante disponer de un conjunto de datos mínimos del paciente mediante un soporte informático que permita a todos los profesionales disponer en todo momento de datos relevantes de la historia clínica tanto de AP como del hospital.

Es curioso observar cómo información muy relevante no es aportada por la familia por ignorancia, despiste o por no querer revelar diagnósticos molestos o dolorosos, como si quisieran borrarlos de su memoria. Lo más probable es que unas creencias erróneas acerca de la enfermedad hayan llevado a omitir la información necesaria y a no considerar tan importante el tratamiento, por lo que hubiera sido muy conveniente explorar estas creencias que tan determinantes son para el correcto cumplimiento del tratamiento ${ }^{1}$.

Ante una situación de posible incumplimiento de un tratamiento importante es necesaria la intervención del equipo de AP. El enfoque será siempre positivo: intentar motivar, responsabilizar y ofrecer información sobre la enfermedad y sus repercusiones en la comunidad y en la escuela. Eventualmente, puede ser necesaria la intervención de otros profesionales de trabajo social, incluso de vigilancia epidemiológica ${ }^{2}$.

Por otra parte, la posibilidad de observación frecuente que tienen el médico y el pediatra de AP han permitido en este caso no solamente replantear el diagnóstico y solicitar las pruebas oportunas del hospital, sino también sospechar el incumplimiento del tratamiento. De esta manera, sin la colaboración entre ambos niveles no se hubiera podido dar una sistencia correcta. El hospital aporta en este caso tecnología diagnóstica, PCR, detección de sensibilidad genética y mejoras en los cultivos, así como conocimientos y experiencia en el uso de antituberculosos, y AP aporta datos de la historia que han permitido replantear el diagnóstico y sospechar el incumplimiento del tratamiento. Es importante que los profesionales de los respectivos niveles conozcan las guías y protocolos de actuación que permitan una atención continuada y de calidad ${ }^{3-5}$.

Es interesante también observar cómo determinadas pruebas pueden ser un factor de confusión. En 
este caso la IgM positiva para Mycoplasma orientó el diagnóstico inicial en otra dirección. La única explicación que le encontramos a este fenómeno es la posible coinfección con Mycoplasma pneumoniae como oportunista, hecho constatado en la bibliografía ${ }^{6,7}$.

A la hora de cambiar el diagnóstico de neumonía atípica por el de tuberculosis, resultó de utilidad para los autores plantearse al menos estas cuatro preguntas: si estoy en el diagnóstico correcto, ¿qué debo esperar que ocurra?, ¿cómo voy a saber si es-

\section{BIBLIOGRAFÍA}

1. Bimbela Pedrola JL. No puedo entenderlo, ahora que hay medicación no se la toma. FMC. 2001;8:551-2.

2. Fuentes Vigil J, Alonso Arias S. El cumplimiento terapéutico. FMC. 2000;07:386-7.

3. Grupo de trabajo de la Guía de Práctica Clínica sobre el diagnóstico, el tratamiento y la prevención de la tuberculosis. Centro Cochrane Iberoamericano, coordinador. Guía de Práctica Clínica sobre el diagnóstico, el tratamiento y la prevención de la tuberculosis. Plan de Calidad para el Sistema Nacional de Salud del Ministerio de Sanidad, Política Social e Igualdad. Agència d'Informació, Avaluació i Qualitat en Salut (AIAQSS) de Cataluña; 2009. Guías de Práctica Clínica en el SNS: AATRM № 2007/26.

4. Zafra Anta MA, Sánchez Vicente Al, Esteban Peris A. Tuberculosis (v.1/2007). Guía_ABE. Infecciones en Pediatría. Guía rápida para la selección del tratamiento antimicrobiano empírico [en línea] [actuali- toy equivocado?, ¿qué puede ocurrir si estoy equivocado?, y ¿qué voy a hacer posteriormente? ${ }^{8}$.

\section{CONFLICTO DE INTERESES}

Los autores declaran no presentar conflictos de intereses en relación con la preparación y publicación de este artículo.

\section{ABREVIATURAS}

AP: Atención Primaria • LSD: lóbulo superior derecho • PCR: reacción en cadena de la polimerasa • PTB: prueba de tuberculina.

zado el 01/05/2007; consultado el 10/01/2013]. Disponible en www.guia-abe.es

5. Moreno-Pérez D, Andrés Martín A, Altet Gómez N, Baquero-Artigao F, Escribano Montaner A, GómezPastrana Durán D, et al. Diagnóstico de la tuberculosis en la edad pediátrica. Documento de Consenso de la Sociedad Española de Infectología Pediátrica (SEIP) y la Sociedad Española de Neumología Pediátrica (SENP). An Pediatr (Barc). 2010;73:143e1-e4.

6. Hussels $\mathrm{H}$, Heidbüchel P, Lohding M. Pulmonary infection with Mycoplasma pneumoniae and coincidently isolation of Mycobacterium fortuitum from sputum. Praxis und Klinik der Pneumologie. 1980; 34(4):225-8

7. Skriagina EM, Kolomiets AG, Grits MA, Lukianenko IG, Kolomiets ND, Gurevich GL. Tuberculosis and opportunistic infections. Probl Tuberk. 1999;(6):25-6

8. Alonso Arias S, Fuentes VigilL J. La red de seguridad. Imprescindible para las acrobacias diagnósticas en Atención Primaria. FMC. 2000;07:609-10. 